\title{
Keldysh and Doi-Peliti Techniques for out-of-Equilibrium Systems.
}

\author{
Alex Kamenev \\ Department of Physics, Technion, Haifa 32000, Israel.
}

(February 1, 2008)

\begin{abstract}
Lecture notes presented at Windsor NATO school on "Field Theory of Strongly Correlated Fermions and Bosons in Low-Dimensional Disordered Systems" (August 2001). The purpose of these lectures is to give a brief modern introduction to Keldysh non-equilibrium field theory and its classical analog - Doi-Peliti technique. The special emphasis is put on stressing the analogy between the two approaches.
\end{abstract}

\section{QUANTUM KELDYSH FORMALISM}

1. A bit of history. The name of the technique takes its origin in 1964 paper of L. V. Keldysh [1]. Among the earlier closely related approaches, one should mention J. Schwinger [2] and R. P. Feynman and F. L. Vernon [3]. Classical counterparts of the Keldysh technique are extremely useful and interesting on their own. I shall mention Wyld diagrammatic technique [7], which is the basis of the modern theory of turbulence; Martin-Siggia-Rose-DeDominics method [8] for stochastic systems; and Doi-Peliti technique [9]10] for reaction-diffusion systems and cellular automata. Despite the unique power and wide popularity of the technique its pedagogical presentations are rare. One may find some in books [4,5] or in a review [6]. This lecture is not an alternative for the existing literature. Using the simplest possible example, I try to explain the general structure of the theory, its relation to other approaches, its potential for novel applications and open problems. Technicalities of the numerous practical applications may be found in the literature and left outside the scope of the lecture. I tried hard, however, to mention possible traps and silent points which are rarely exposed explicitly.

2. Motivation. The Keldysh formulation of the many-body theory is useful in the following cases:

- For treatment of systems not in the thermal equilibrium [1].

- For calculation of the full counting statistics of a quantum mechanical observable (as opposed to an average value or correlators) [11, 12,

- As an alternative to the replica and supersymmetry methods in the theory of disordered and glassy systems [13 [17.

- For equilibrium problems where Matsubara analytical continuation may prove to be sophisticated [6].

3. Closed time contour. The standard construction of the zero temperature (or equilibrium) many-body theory (see e.g. [18,5]) involves the procedure of adiabatic switching interactions on at distant past and then switching it off at distant future. The crucial assumption here is that starting from the ground (or equilibrium) state of the system at $t=-\infty$ one arrives to the same state at $t=+\infty$ (acquiring some phase factor along the way). This is clearly not the case out of the equilibrium. Starting from some arbitrary distribution and then switching interactions on and off, one is going to find the system in some unpredictable state. The latter depends, in general, on the peculiarities of the switching procedure. The absence of knowledge about the finite state spoils completely the entire construction [18, 20]. One would like thus to build a theory which avoids reference to the state at $t=+\infty$. We still need to know a finite state (since we calculate traces). The Schwinger suggestion is to make the finite state to be exactly the initial one. The central idea is to let a quantum system to evolve first in the forward direction in time and then to "rewind" its evolution back, playing the movie in the backward direction. One ends up thus with the need to construct a theory with the time evolution along the two-branch contour depicted on Fig. 1. Then, no matter what was the state at $t=+\infty$, after the backward evolution it will return back to the known initial state. In this construction there is no 
switching of interactions in the future. Both switchings on and off take place in the past: on - on the forward branch and off - on the backward. How to construct such a theory and how to use it - is the subject of this chapter.

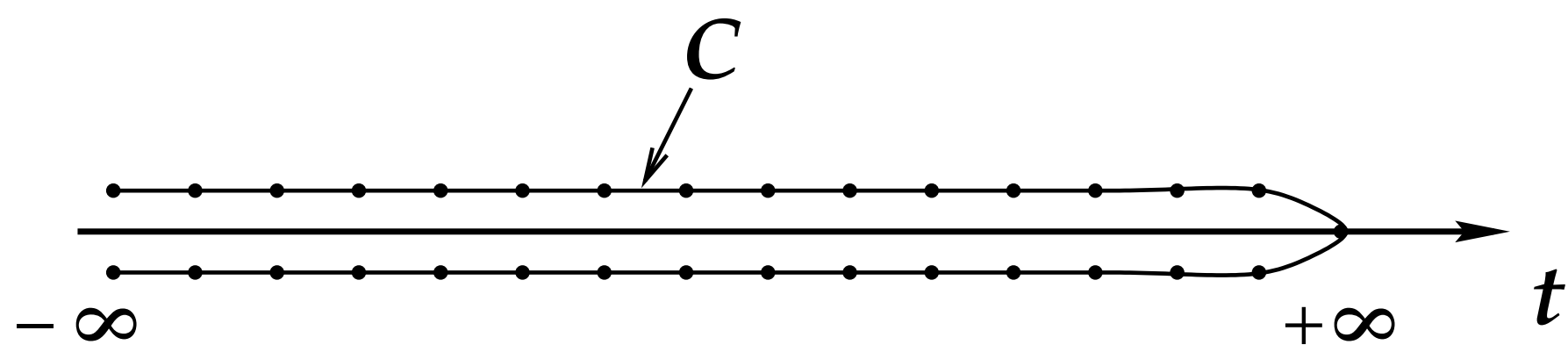

FIG. 1 The closed Keldysh time contour $\mathcal{C}$. Dots on the forward and the backward branches of the contour denote the discretized time points.

4. Field theory. To be specific, let me consider the simplest possible many-body system. It consists of bosons living in a single quantum state with the energy $\omega_{0}$ :

$$
H=\omega_{0} a^{\dagger} a
$$

here $a^{\dagger}$ and $a$ are bosonic creation and annihilation operators. Let us consider the "partition function" defined as

$$
Z=\operatorname{Tr}\left\{\rho_{0} U_{\mathcal{C}}\right\} / \operatorname{Tr}\left\{\rho_{0}\right\}
$$

where $U_{\mathcal{C}}$ is the evolution operator along the closed contour $\mathcal{C}$. If we assume (as we shall do for a while) that all the external fields are exactly the same on the forward and backward branches of the contour, then $U_{\mathcal{C}}=1$ and therefore $Z=1$. In Eq. (2) $\rho_{0}=\rho(H)$ is some 円 density matrix operator defined at $t=-\infty$. In our example $\operatorname{Tr}\left\{\rho_{0}\right\}=\left[1-\rho\left(\omega_{0}\right)\right]^{-1}$. An important point is that in general $\operatorname{Tr}\left\{\rho_{0}\right\}$ is an interaction- and disorder-independent constant. Indeed both interactions and disorder are supposed to be switched on (and off) on the forward (backward) parts of the contour sometime after (before) $t=-\infty$. I shall therefore frequently omit this constant or refer to it as being unity - it never causes a confusion.

The next step is to divide the $\mathcal{C}$ contour into $2 N+1$ time steps of the length $\delta_{t}$, such that $t_{1}=t_{2 N+1}=-\infty$ and $t_{N+1}=+\infty$ as shown on Fig. 11. Following the standard route [19], we obtain the coherent state functional integral, by introducing a resolution of unity at each time step. Taking the $N \rightarrow \infty$ limit we obtain for the partition function

$$
Z=\frac{1}{\operatorname{Tr}\left\{\rho_{0}\right\}} \int \mathcal{D} \bar{\phi} \phi \exp \{i S[\bar{\phi}(t), \phi(t)]\},
$$

where the action is given by

$$
S[\bar{\phi}(t), \phi(t)]=\int_{\mathcal{C}} d t \bar{\phi}(t) \mathcal{D}^{-1} \phi(t)
$$

and $\mathcal{D}^{-1}=\left[i \partial_{t}-\omega_{0}\right]$. It is important to remember that this continuous notation is only a short way to represent the $2 N \times 2 N$ matrix $\mathcal{D}_{i j}^{-1}$

\footnotetext{
${ }^{1}$ To accommodate the equilibrium initial density matrix, $\rho_{0}=\exp \{-\beta(H-\mu N)\}$, it is sometimes suggested to add an imaginary (vertical) part to the Keldysh contour at $t=-\infty$. To my opinion this procedure only obscures the structure of the theory. Indeed, the technique is not restricted in any way to the initial distribution being the equilibrium one. Therefore it is much preferred to keep the structure general and not commit oneself with unnecessary assumptions.
} 


$$
-i \mathcal{D}_{i j}^{-1}=\left[\begin{array}{ccccccc}
1 & 0 & & & & & \\
-1+\delta_{t} i \omega_{0} & 1 & 0 & & & & \\
& -1+\delta_{t} i \omega_{0} & 1 & 0 & & & \\
& & -1+\delta_{t} i \omega_{0} & 1 & 0 & & \\
& & \ddots & \ddots & \ddots & & \\
& & & & & & \\
& & & & & \left.-1-\delta_{t} i \omega_{0}\right) \rho\left(\omega_{0}\right) & \\
& & & & & -1-\delta_{t} i \omega_{0} & 1
\end{array}\right]
$$

The change of sign $+\delta_{t} \rightarrow-\delta_{t}$ takes place in the center of the matrix at the point where the time axis changes its direction. It is straightforward to evaluate the determinant of such matrix

$$
\operatorname{det}\left[-i \mathcal{D}^{-1}\right]=1+(-1)^{2 N+1} \rho\left(\omega_{0}\right)\left(-1+\delta_{t} i \omega_{0}\right)^{N}\left(-1-\delta_{t} i \omega_{0}\right)^{N} \rightarrow 1-\rho\left(\omega_{0}\right) ;
$$

Recalling that $\rho\left(\omega_{0}\right)<1$, one may convince himself that all eigenvalues of the matrix $-i \mathcal{D}_{i j}^{-1}$ have positive real part. This fact secures convergence of the functional integral. Performing the integration, one not-surprisingly obtains for the partition function

$$
Z=\frac{\operatorname{det}^{-1}\left[-i \mathcal{D}^{-1}\right]}{\operatorname{Tr}\left\{\rho_{0}\right\}}=1
$$

Seemingly absent in the continious notations - the upper right element of the matrix is extremely important to obtain the correct normalization. It is not less important to find the proper correlation functions.

We divide next the bosonic field $\phi(t)$ onto the two components $\phi_{f}(t)$ and $\phi_{b}(t)$ which reside on the forward and the backward parts of the time contour correspondingly. The action may be now rewritten as $S\left[\phi_{f}\right]-S\left[\phi_{b}\right]$, with the time integration running along the real axis (the minus sign comes from reversing direction of the time integration on the backward part of the contour).

5. Correlation functions. Now comes the most unpleasant part: to invert the matrix and find the correlation (or Green) functions. We may save ourselves a lot of efforts by recalling that the functional integral always produces the time-ordered Green functions [19] and that the time arguments on the backward branch are always after those on the forward. This way one finds

$$
\begin{aligned}
-i\left\langle\phi_{f}(t) \bar{\phi}_{f}\left(t^{\prime}\right)\right\rangle & =\mathcal{D}^{T}\left(t, t^{\prime}\right)=\theta\left(t-t^{\prime}\right) \mathcal{D}^{>}\left(t, t^{\prime}\right)+\theta\left(t^{\prime}-t\right) \mathcal{D}^{<}\left(t, t^{\prime}\right) ; \\
-i\left\langle\phi_{f}(t) \bar{\phi}_{b}\left(t^{\prime}\right)\right\rangle & =\mathcal{D}^{<}\left(t, t^{\prime}\right)=\left(-i n e^{-i \omega_{0}\left(t-t^{\prime}\right)}\right) ; \\
-i\left\langle\phi_{b}(t) \bar{\phi}_{f}\left(t^{\prime}\right)\right\rangle & =\mathcal{D}^{>}\left(t, t^{\prime}\right)=\left(-i(n+1) e^{-i \omega_{0}\left(t-t^{\prime}\right)}\right) ; \\
-i\left\langle\phi_{b}(t) \bar{\phi}_{f}\left(t^{\prime}\right)\right\rangle & =\mathcal{D}^{\tilde{T}}\left(t, t^{\prime}\right)=\theta\left(t^{\prime}-t\right) \mathcal{D}^{>}\left(t, t^{\prime}\right)+\theta\left(t-t^{\prime}\right) \mathcal{D}^{<}\left(t, t^{\prime}\right),
\end{aligned}
$$

where the angular brackets are understood as the functional integration with the action given above. The structure is general, but $\mathcal{D}^{>}=-i\left\langle\left|a(t) a^{\dagger}\left(t^{\prime}\right)\right|\right\rangle$ and $\mathcal{D}^{<}=-i\left\langle\left|a^{\dagger}\left(t^{\prime}\right) a(t)\right|\right\rangle$ are given for orientation in brackets for our simple example $\left(n=\rho\left(\omega_{0}\right) /\left(1-\rho\left(\omega_{0}\right)\right)\right.$ is the bosonic occupation number $)$.

It may be surprising to see the non-zero off-diagonal correlators $\left\langle\phi_{f} \bar{\phi}_{b}\right\rangle$ and $\left\langle\phi_{b} \bar{\phi}_{f}\right\rangle$, while the action seems to be perfectly diagonal in the $f-b$ space. The trick is exactly in the presence of the upper-right element of the discretized matrix, hidden in the continuous representation. Notice, that the presence of the off-diagonal elements does not contradict to the continuous notations. Indeed, $\left[i \partial_{t}-\omega_{0}\right] \mathcal{D}^{>,<}=0$, while $\left[i \partial_{t}-\omega_{0}\right] \mathcal{D}^{T, \tilde{T}}= \pm \delta\left(t-t^{\prime}\right)$. Therefore in

\footnotetext{
${ }^{2}$ The more familiar definition of the Green functions is $\mathcal{D}=-i\langle\phi \bar{\phi}\rangle / Z$, since in our case $Z=1-$ we are doing OK.
} 
the obvious $2 \times 2$ matrix notations $\mathcal{D}^{-1} \mathcal{D}=1$, as it should be. The point is that the $\left[i \partial_{t}-\omega_{0}\right]^{-1}$ operator is not uniquely defined. The boundary conditions must be specified and the upper right element does exactly this.

Obviously not all four Green functions defined above are independent. Indeed, an inspection shows that

$$
\mathcal{D}^{T}+\mathcal{D}^{\tilde{T}}=\mathcal{D}^{>}+\mathcal{D}^{<} .
$$

One would therefore like to perform a linear transformation of the fields to benefit explicitly from this relation. This is achieved by the Keldysh rotation.

5. Keldysh rotation. Let us define the new fields as

$$
\phi_{c l}(t)=\frac{1}{2}\left(\phi_{f}(t)+\phi_{b}(t)\right) ; \quad \phi_{q}(t)=\frac{1}{2}\left(\phi_{f}(t)-\phi_{b}(t)\right)
$$

with the analogous transformation for the conjugated fields. The integration measure changes by unessential multiplicative constant. The subscripts $c l$ and $q$ stand for classical and quantum components of the fields correspondingly. The rational for such notations will become clear shortly. First, a little algebra shows that 3

$$
-i\left\langle\phi_{\alpha}(t) \bar{\phi}_{\beta}\left(t^{\prime}\right)\right\rangle=\frac{1}{2}\left(\begin{array}{cc}
\mathcal{D}^{K}\left(t, t^{\prime}\right) & \mathcal{D}^{R}\left(t, t^{\prime}\right) \\
\mathcal{D}^{A}\left(t, t^{\prime}\right) & 0
\end{array}\right)
$$

where $\alpha, \beta=c l, q$. Here indexes $R, A, K$ stay for retarded, advanced and Keldysh components of the Green function. These three are the main objects of the Keldysh technique. They are defined as

$$
\begin{aligned}
& \mathcal{D}^{R}=\mathcal{D}^{T}-\mathcal{D}^{<}=\theta\left(t-t^{\prime}\right)\left(\mathcal{D}^{>}-\mathcal{D}^{<}\right) ; \\
& \mathcal{D}^{A}=\mathcal{D}^{T}-\mathcal{D}^{>}=\theta\left(t^{\prime}-t\right)\left(\mathcal{D}^{<}-\mathcal{D}^{>}\right) ; \\
& \mathcal{D}^{K}=\mathcal{D}^{>}+\mathcal{D}^{<}
\end{aligned}
$$

Notice that the retarded (advanced) component is lower (upper) triangular matrix in the time space. Moreover, our regularization of the functional integral guarantees that these functions never appear at exactly coinciding arguments, removing thus uncertainty about the value $\theta(t-t)=\theta(0)$. Fields $\bar{\phi}$ always appear at one time step $\delta_{t}$ after $\phi$ fields on the Keldysh contour. As a result, $\bar{\phi}_{f}(t)$ is after $\phi_{f}(t)$ and $\bar{\phi}_{b}(t)$ is before $\phi_{b}(t)$. Employing Eqs. (8) and (12), one may see that with this convention $\mathcal{D}^{R}(t, t) \equiv \mathcal{D}^{R}\left(t, t+\delta_{t}\right)=0$ and $\mathcal{D}^{A}(t, t) \equiv \mathcal{D}^{A}\left(t+\delta_{t}, t\right)=0$. In the continuous notations it is equivalent to the choice of the regularization, where $\theta(0)=0$. As a result, for any number $t$ of retarded or advanced functions one has

$$
\begin{aligned}
& \operatorname{Tr}\left\{\mathcal{D}^{R} \mathcal{D}^{R} \ldots \mathcal{D}^{R}\right\}=0 \\
& \operatorname{Tr}\left\{\mathcal{D}^{A} \mathcal{D}^{A} \ldots \mathcal{D}^{A}\right\}=0
\end{aligned}
$$

where the traces are understood as time integrations. If a problem is translationally invariant in time - the same statement has the simple reinterpretation

$$
\int_{-\infty}^{\infty} d \omega \mathcal{D}^{R}(\omega) \mathcal{D}^{R}\left(\omega+\Omega_{1}\right) \ldots \mathcal{D}^{R}\left(\omega+\Omega_{n}\right)=0
$$

\footnotetext{
${ }^{3}$ For fermions it is more convenient to transform $\psi$ and $\bar{\psi}$ in the different manner (it is allowed since $\psi$ and $\bar{\psi}$ are not actually complex conjugated, but rather notations). As a result, the standard matrix form of the fermionic propagator is

$$
\left(\begin{array}{cc}
\mathcal{G}^{R}\left(t, t^{\prime}\right) & \mathcal{G}^{K}\left(t, t^{\prime}\right) \\
0 & \mathcal{G}^{A}\left(t, t^{\prime}\right)
\end{array}\right)
$$

In this notation fermion-boson interactions take an especially simple form $S_{\text {int }} \sim \phi_{\alpha} \bar{\psi} \gamma^{\alpha} \psi$, with $\gamma^{\text {cl }}=1$ and $\gamma^{q}=\sigma_{1}$ 15. Since fermions are never a classical field, this form of the fermionic propagator is not related to our subsequent discussion.

${ }^{4}$ This is true for a single function as well. For example, if $\mathcal{D}^{R}=\left(D q^{2}-i \omega\right)^{-1}$, one may worry that $\int d \omega\left(D q^{2}-i \omega\right)^{-1}$ is divergent. This is not the case, however, since this integral is simply $\mathcal{D}^{R}(t, t)=0$.
} 
which is an obvious consequence of retarded Green function being an analytic function in the entire upper half plane of complex energy. From Eq. (11) it is obvious that

$$
\mathcal{D}^{A}=\left[\mathcal{D}^{R}\right]^{\dagger},
$$

where Hermit conjugation includes transposition of the time arguments.

In our simple example $\mathcal{D}^{>}=-i(n+1) e^{-i \omega_{0}\left(t-t^{\prime}\right)}$ and $\mathcal{D}^{<}=-i n e^{-i \omega_{0}\left(t-t^{\prime}\right)}$, where $n=n\left(\omega_{0}\right)=\rho\left(\omega_{0}\right) /\left(1-\rho\left(\omega_{0}\right)\right)$ is the bosonic occupation number (since the system is non-interacting the initial distribution function does not evolve). As a result

$$
\begin{aligned}
& \mathcal{D}^{R}\left(t, t^{\prime}\right)=-i \theta\left(t-t^{\prime}\right) e^{-i \omega_{0}\left(t-t^{\prime}\right)} \\
& \mathcal{D}^{A}\left(t, t^{\prime}\right)=i \theta\left(t^{\prime}-t\right) e^{-i \omega_{0}\left(t-t^{\prime}\right)} \\
& \mathcal{D}^{K}\left(t, t^{\prime}\right)=-i\left(2 n\left(\omega_{0}\right)+1\right) e^{-i \omega_{0}\left(t-t^{\prime}\right)}
\end{aligned}
$$

Notice that the retarded and advanced components are independent on the distribution function, whereas the Keldysh component does depend on it. This statement is much more general than our toy example. Going to the frequency representation, one finds that $\mathcal{D}^{K}(\omega)=-2 \pi i\left(2 n\left(\omega_{0}\right)+1\right) \delta\left(\omega-\omega_{0}\right)=-2 \pi i(2 n(\omega)+1) \delta\left(\omega-\omega_{0}\right)$. In the particular case of the thermal equilibrium, one has

$$
\mathcal{D}^{K}(\omega)=\operatorname{coth} \frac{\beta \omega}{2}\left(\mathcal{D}^{R}(\omega)-\mathcal{D}^{A}(\omega)\right) .
$$

The last equation constitutes the statement 5 of the fluctuation-dissipation theorem (FDT). It implies (as we shall see below) the rigid relation between response and correlation functions at equilibrium. FDT is always satisfied in the thermal equilibrium even in the presence of the interactions. We shall see later why the interactions do not change this relation. In general case it is convenient to define a function (matrix in the time space) $F$ via

$$
\mathcal{D}^{K}=\mathcal{D}^{R} F-F \mathcal{D}^{A}
$$

and refer to $F$ (or better its Wigner transform) as the distribution function. At equilibrium $F\left(t-t^{\prime}\right) \rightarrow F(\omega)=$ $\operatorname{coth} \frac{\beta \omega}{2}=2 n(\omega)+1$, where $n(\omega)$ is the Planck distribution.

6. Keldysh action and causality. Once we have understood the structure of the propagator in the $c l-q$ space, we may go back to the action. Inverting the matrix Eq. (11), one finds 6

$$
S\left[\phi_{c l}, \phi_{q}\right]=2 \iint_{-\infty}^{\infty} d t d t^{\prime}\left(\bar{\phi}_{c l}(t), \bar{\phi}_{q}(t)\right)\left(\begin{array}{cc}
0 & {\left[\mathcal{D}^{A}\right]^{-1}} \\
{\left[\mathcal{D}^{R}\right]^{-1}} & {\left[\mathcal{D}^{-1}\right]^{K}}
\end{array}\right)_{\left(t, t^{\prime}\right)}\left(\begin{array}{c}
\phi_{c l}\left(t^{\prime}\right) \\
\phi_{q}\left(t^{\prime}\right)
\end{array}\right)
$$

where $\left[\mathcal{D}^{-1}\right]^{K}=\left[\mathcal{D}^{R}\right]^{-1} F-F\left[\mathcal{D}^{A}\right]^{-1} \neq\left[\mathcal{D}^{K}\right]^{-1}$. Of course, in our simple example $\left[\mathcal{D}^{R, A}\right]^{-1}=\delta\left(t-t^{\prime}\right)\left[i \partial_{t^{\prime}}-\omega_{0} \pm i 0\right]$ and $\left[\mathcal{D}^{-1}\right]^{K}=0$ and therefore the correlator matrix is basically $\delta\left(t-t^{\prime}\right)\left[i \partial_{t^{\prime}}-\omega_{0}\right] \sigma_{1}$, as it should be. The point is that the structure of the Gaussian action given by Eq. (19) is absolutely generic and correctly encodes regularization of the functional integral. Since the Keldysh component carries information about the density matrix, there is no need any more to remember about the discreet representation. As we shall see below this structure remains intact under any perturbative renormalization. For the lack of a better terminology we shall refer to the robustness of this structure as the causality principle 117] (since it is the reason for the response functions being casual, see below). Let us devote some time to this structure.

\footnotetext{
${ }^{5}$ The analogous statement for fermions reads as

$$
\mathcal{G}^{K}(\epsilon)=\tanh \frac{\beta \epsilon}{2}\left(\mathcal{G}^{R}(\epsilon)-\mathcal{G}^{A}(\epsilon)\right)
$$

${ }^{6}$ Notice that the matrix in the exponent of the functional integral, $i \mathcal{D}^{-1}$, is anti-Hermitian ! The convergence factors are hidden in the regularization.
} 
Consider first the $c l-c l$ element, which is zero. This zero may be traced back to the relation (9). It has, however, the much simpler interpretation. It says that if one takes a purely classical field configuration, $\phi_{q}=0$, the action is zero. But this is obvious, since in this case $\phi_{f}=\phi_{b}$ and the action on the forward contour cancels exactly that on the backward. We therefore arrive to the simple but extremely important statement:

$$
S\left[\phi_{c l}, \phi_{q}=0\right]=0 .
$$

From this perspective the relation (9) is simply one of manifestations of the causality. Obviously Eq. (20) is not restricted to the Gaussian action. Consider e.g. the simplest interaction action $S_{\text {int }}=\frac{\lambda}{2} \int_{\mathcal{C}} d t(\bar{\phi} \phi)^{2}=\frac{\lambda}{2} \int d t\left[\left(\phi_{f} \phi_{f}\right)^{2}-\right.$ $\left.\left(\bar{\phi}_{b} \phi_{b}\right)^{2}\right]$. Notice that this is the continuous notation; in discreet version $\bar{\phi}$ fields on the Keldysh contour $\mathcal{C}$ are taken at one step $\delta_{t}$ later time with respect to $\phi$ fields. Transforming to the classical-quantum components, one finds

$$
S_{i n t}\left[\phi_{c l}, \phi_{q}\right]=4 \lambda \Re \int_{-\infty}^{\infty} d t \bar{\phi}_{q} \bar{\phi}_{c l}\left(\phi_{c l}^{2}+\phi_{q}^{2}\right),
$$

which obviously satisfy Eq. (20).

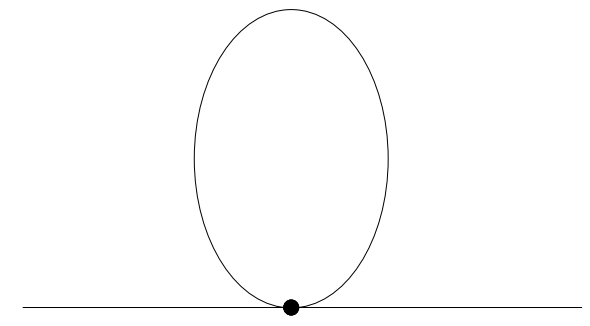

a)

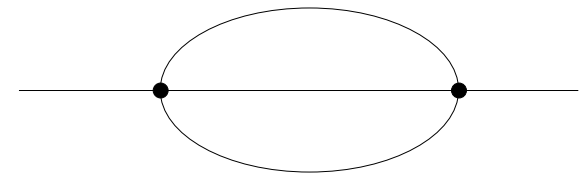

b)

FIG. 8 First and second order

diagrams for the $(\bar{\phi} \phi)^{2}$ theory.

The fact that $c l-q$ and $q-c l$ components in Eq. (19) are advanced and retarded (means triangular in the time representation) is of the fundamental importance as well. Due to this fact Eq. 20) is intact in any order of the perturbation theory. Consider, for example, the first order correction in $\lambda$ to the $c l-c l$ element of the Gaussian action (which is zero). The corresponding diagram is depicted on Fig. 2a. From Eq. (21) one reads out

$$
\delta S_{c l-c l}=4 \lambda \Re \int_{-\infty}^{\infty} d t \bar{\phi}_{c l}(t) \mathcal{D}^{R}(t, t) \phi_{c l}(t) .
$$

As was explained above, $\mathcal{D}^{R}(t, t)=0$, and the $c l-c l$ element obviously remains zero. Employing Eqs. (13), (14) one may show that all higher loop corrections do not change this fact. One may say that Eqs. (13), (14) are part of the causality principle. To complete the prove that causality is preserved in perturbation theory, one has to show that $c l-q$ and $q-c l$ components remain purely advanced and retarded correspondingly. A reader is advised to check that this is indeed the case in the second order, Fig. 2 b.

7. Saddle point equations. Before doing the perturbation theory we have to discuss saddle points of the action. Eq. (20) states that there are no terms in the action which have zero power of both $\bar{\phi}_{q}$ and $\phi_{q}$. The same is obviously true regarding $\delta S / \delta \bar{\phi}_{c l}$ and therefore the saddle point equation

$$
\frac{\delta S}{\delta \bar{\phi}_{c l}}=0
$$

may be always solved by

$$
\Phi_{q}=0 .
$$


The saddle point solutions are denoted by capital $\Phi$. One may check that this is indeed the case for the action given by Eqs. (19), 21). Under the condition Eq. (24) the second saddle point equation takes the form

$$
\frac{1}{2} \frac{\delta S}{\delta \bar{\phi}_{q}}=\mathcal{O}^{R}\left[\Phi_{c l}\right] \Phi_{c l}=0
$$

where $\mathcal{O}^{R}\left[\Phi_{c l}\right]$ is retarded operator describing the classical dynamics of the field. In our example

$$
\mathcal{O}^{R}\left[\Phi_{c l}\right]=i \partial_{t}-\omega_{0}+\lambda\left|\Phi_{c l}\right|^{2}
$$

and the saddle point equation (25) is the time-dependent "Gross-Pitaevskii" equation. We have arrived to the conclusion that among possible saddle point solutions of the Keldysh action, there are always some with zero quantum component and the classical component obeying the classical equation of motion. We shall call such saddle points classical or casual (since perturbation theory on top of a classical solution preserves the causality structure). Notice, that the classical equation of motion is result of variation over the quantum component (in the limit where the later is zero).

In view of Eqs. (20) and (24), the action on any classical solution is zero 1. Recall now that we do the theory for $Z=1$ - this is the exact relation. To reproduce it in the saddle point approximation one has to show that the fluctuation determinant on top of the classical saddle point is unity. It may be a formidable task to demonstrate it explicitly, but it is helpful to remember that this must be the case.

An extremely interesting issue is an existence and role of non-classical saddle point solutions, which have $\Phi_{q} \neq 0$. Such solutions, if exist, have, in general, a non-zero action and may be loosely called instantons. Examples of instantons include (thermal) escape from a quantum well [20] and Wigner-Dyson statistics in metal grains [17]. Since the normalization $Z=1$ is entirely due to classical saddle points, instantons must have strictly zero contribution to the partition function, $Z$. They may, however, contribute to correlation and response functions. The subject is poorly developed (unlike instantons in the imaginary time) and requires much more attention.

8. Semiclassical approximation. Let me concentrate now on a classical saddle point, Eqs. (24), (25). To include quantum effects in the semiclassical approximation one has to include fluctuations of the $\phi_{q}$ field near zero. To this end we keep terms up to the second order in $\phi_{q}$ in the action. The resulting semiclassical action takes the following general form

$$
S_{s c l}=2 \iint_{-\infty}^{\infty} d t d t^{\prime}\left[\bar{\phi}_{q}\left[\mathcal{D}^{-1}\right]^{K} \phi_{q}+\left(\bar{\phi}_{q} \mathcal{O}^{R}\left[\phi_{c l}\right] \phi_{c l}+c . c .\right)\right]
$$

c.c. stays for the complex conjugation. From this point one may proceed in two directions:

(i) Since the action Eq. (27) is Gaussian in $\phi_{q}$ one may integrate it out and end up with the theory of the single filed, $\phi_{c l}$. The corresponding action is

$$
S_{s c l}\left[\phi_{c l}\right]=2 \iint_{-\infty}^{\infty} d t d t^{\prime} \bar{\phi}_{c l}\left(\mathcal{O}^{A}\left[\bar{\phi}_{c l}\right] \mathcal{D}^{A}\right)\left[\mathcal{D}^{K}\right]^{-1}\left(\mathcal{D}^{R} \mathcal{O}^{R}\left[\phi_{c l}\right]\right) \phi_{c l}
$$

If the non-linearity in $\phi_{c l}$ is neglected, one ends up with the Gaussian theory governed by the inverse Keldysh Green function, $S \sim \bar{\phi}_{c l}\left[\mathcal{D}^{K}\right]^{-1} \phi_{c l}$ \&. In general, the action Eq. (28) is pretty complicated. If it contains only a finite number of the degrees of freedom and is short-ranged in time, one may use the transfer-matrix technique to write down the corresponding "Schrödinger" equation. The result is the Focker-Planck equation.

\footnotetext{
${ }^{7}$ This statement is a close relative of the Parisi-Efetov-Wegner theorem in supersymmetry or the fact that a replica symmetric saddle point results in zero action in the replica limit.

${ }^{8}$ If $\mathcal{D}^{R, A}=(0 \mp i \omega)^{-1}$, then in the thermal equilibrium (c.f. Eq. (17)), $\left[\mathcal{D}^{K}\right]^{-1}=\frac{-i}{2} \omega \tanh \frac{\beta \omega}{2}$. In the $T=0$ limit one obtains the non-local quantum dissipative action, $S \sim|\omega|\left|\phi_{c l}(\omega)\right|^{2}$, while in the high temperature regime the action is local $S \sim \beta\left|\dot{\phi}_{c l}(t)\right|^{2}$.
} 
(ii) One may perform the Hubbard-Stratonovich transformation with the auxiliary stochastic field $\xi(t)$ to decouple the quadratic term in Eq. (27). The resulting action is linear in $\phi_{q}$. Therefore the integration over $\phi_{q}$ leads to the functional $\delta$-function. As a result one obtains stochastic Langevin equation 9

$$
\mathcal{O}^{R}\left[\phi_{c l}\right] \phi_{c l}(t)=\xi(t)
$$

where $\xi(t)$ is a Gaussian noise with the correlator

$$
\left\langle\xi(t) \bar{\xi}\left(t^{\prime}\right)\right\rangle=\frac{i}{2}\left[\mathcal{D}^{-1}\right]^{K}\left(t, t^{\prime}\right) .
$$

For the diffusive dynamics, $\left[\mathcal{D}^{R, A}\right]^{-1}=D \nabla^{2} \mp i \omega$, in the thermal equilibrium $i\left[\mathcal{D}^{-1}\right]^{K}(\omega)=i \operatorname{coth} \frac{\beta \omega}{2}\left(\left[\mathcal{D}^{R}\right]^{-1}(\omega)-\right.$ $\left.\left[\mathcal{D}^{A}\right]_{-}^{-1}(\omega)\right) \rightarrow 4 T$ in the limit $\omega \ll T$. In this case one obtains the classical Langevin force with the correlator $\left\langle\xi(t) \bar{\xi}\left(t^{\prime}\right)\right\rangle=2 T \delta\left(t-t^{\prime}\right)$. The opposite limit is called sometimes the quantum Langevin equation.

Notice that the above procedure may be reversed. Start from the classical Langevin equation, then introduce functional $\delta$-function. Finally exponentiate it with the auxiliary field called $\phi_{q}$ and integrate out the Gaussian noise. Such procedure is known in the literature as Martin-Siggia-Rose-DeDominics (MSRD) [8] technique. The result is the semi-classical Keldysh action, Eq. (27), in the high temperature limit. In the MSRD literature the field $\phi_{c l}$ is called $\phi$, while $\phi_{q}$ is usually called $\hat{\phi}$. One may wonder if one can form their symmetric and antisymmetric combinations $\phi_{f}=\phi+\hat{\phi}$ and $\phi_{b}=\phi-\hat{\phi}$ to recover an "underlying quantum theory" with the action $S\left[\phi_{f}\right]-S\left[\phi_{b}\right]$. In general it does not work since MSRD is only the semiclassical limit of Keldysh. Therefore terms of the order $O\left(\hat{\phi}^{3}\right)$ may be "missing".

9. FDT and kinetic equation. Having understood the structure of the saddle points, we can construct a perturbative expansion around a classical saddle point 10 . Let say we are about to calculate the full Green function, defined as $\mathcal{D}_{\alpha, \beta}\left(t, t^{\prime}\right)=-2 i\left\langle\phi_{\alpha}(t) \bar{\phi}_{\beta}\left(t^{\prime}\right)\right\rangle$, where $\alpha, \beta=c l, q$. This is done in a standard way by expanding the exponent of the non-Gaussian action and applying the Wick theorem according to Eq. (11). One then rearranges the perturbation theory and defines the irreducible self-energy $\Sigma$ through the relation:

$$
\mathcal{D}=\mathcal{D}_{0}+\mathcal{D}_{0} \Sigma \mathcal{D}_{0}+\mathcal{D}_{0} \Sigma \mathcal{D}_{0} \mathcal{D}_{0} \Sigma \mathcal{D}_{0}+\ldots
$$

where multiplication is understood as the matrix one in the time and $c l-q$ spaces; $\mathcal{D}_{0}$ is the bare propagator, Eq. (11). The causality guarantees (this may be checked order by order) that the self-energy matrix has the structure of the correlator (i.e. $\mathcal{D}_{0}^{-1}$; cf. Eq. (19))

$$
\Sigma_{\alpha, \beta}\left(t, t^{\prime}\right)=\left(\begin{array}{cc}
0 & \Sigma^{A}\left(t, t^{\prime}\right) \\
\Sigma^{R}\left(t, t^{\prime}\right) & \Sigma^{K}\left(t, t^{\prime}\right)
\end{array}\right)
$$

Consider e.g. the second term on the r.h.s. of Eq. (31), after a little algebra one finds

$$
\begin{aligned}
& \delta \mathcal{D} c l, c l=\mathcal{D}_{0}^{K} \Sigma^{A} \mathcal{D}_{0}^{A}+\mathcal{D}_{0}^{R} \Sigma^{R} \mathcal{D}_{0}^{K}+\mathcal{D}_{0}^{R} \Sigma^{K} \mathcal{D}_{0}^{A} \\
& \delta \mathcal{D} c l, q=\mathcal{D}_{0}^{R} \Sigma^{R} \mathcal{D}_{0}^{R} \\
& \delta \mathcal{D} q, c l=\mathcal{D}_{0}^{A} \Sigma^{A} \mathcal{D}_{0}^{A} \\
& \delta \mathcal{D} q, q=0
\end{aligned}
$$

As a result the $q-q$ component remains zero, while $c l-q$ and $q-c l$ remain retarded and advanced correspondingly. Indeed, product of triangular matrices is again a triangular matrix. Obviously, the same is true in all higher orders as well. Therefore the causality structure remains intact even in the presence of non-linearity (at least in perturbation theory). Eq. (31) may be rewritten as the matrix Dyson equation

\footnotetext{
${ }^{9}$ One may worry about appearance of the Jacobean upon integration of the functional $\delta$-function over $\phi_{c l}$. It does not happen with our regularization of the functional integral (Jacobean is unity). The reason is exactly the same which guarantees $Z=1$ upon integration of the fluctuations around a classical saddle point.

${ }^{10} \mathrm{I}$ am not aware about any attempt of doing the perturbation theory on top of a non-classical saddle point.
} 


$$
\left(\mathcal{D}_{0}^{-1}-\Sigma\right) \mathcal{D}=1
$$

It is a simple matter to show that the $q-c l$ component of this equation is nothing but the kinetic equation on the distribution function $F$. Since this procedure is well documented in the literature [1, 4.,6] we shall not describe it here 11. Let me only mention that to obtain the textbook (semi-classical) kinetic equation one has to assume that all external fields are slow on the scale of temperature. This assumption justifies the time-Wigner transform of the matrix $F$, which is a necessary step in the derivation of the kinetic equation.

The other way to obtain the kinetic equation is to introduce time non-local field $Q\left(t, t^{\prime}\right)$, canonically conjugated to the composite operator $\phi(t) \bar{\phi}\left(t^{\prime}\right)$. A small amount of disorder makes $Q\left(t, t^{\prime}\right)$ a dynamic field which is governed by a certain effective action (this action may be obtained by integrating out the fields $\phi$ and $\bar{\phi}$ ). The saddle point equation for this effective action $S_{\text {eff }}[Q]$ is the kinetic equation. As usually, the (semi) classical (kinetic) equation is obtained by variation over the quantum component of the $Q$-field. For the details of this procedure see Ref. 15. One can possibly go beyond the saddle-point approximation by including the Gaussian fluctuations of the quantum component of $Q$ (pretty much in the way we did above). The result of such procedure is probably the Langevin-kinetic equation of Kogan and Shulman [21]. To the best of my knowledge, it was not demonstrated explicitly yet.

10. Sources, external fields, response and correlation functions. Up until now we have been busy with complicated representation of unity: $Z=1$. This is an interesting, but useless job. To make the technique work we need to calculate something more informative. This something is the generating function, which is obtained by adding the source fields to the action. It is convenient to discuss it in parallel with introduction of external classical fields (such as scalar or vector potentials). Let us add the source term $S_{\text {source }}=\frac{1}{2} \int_{\mathcal{C}} d t V \bar{\phi} \phi$ to the action. The source field $V(t)$ is defined on the Keldysh contour, therefore it is rather a couple of fields $\left(V_{f}, V_{b}\right)$, residing on the forward and backward branches correspondingly. We decompose it now on the classical and quantum components exactly in the same way we did it with the $\phi$ field: $V_{c l}=\left(V_{f}+V_{b}\right) / 2$ and $V_{q}=\left(V_{f}-V_{b}\right) / 2$. The source action takes the form

\footnotetext{
${ }^{11}$ The simplest exercise is the theory of the real bosonic field with the interaction $\lambda \phi^{3} \rightarrow 3 \lambda \phi_{c l}^{2} \phi_{q}+\lambda \phi_{q}^{3}$. In the second order the self-energy Fig. 3 is given by $\Sigma^{R, A} \sim \lambda^{2} \mathcal{D}_{0}^{K} * \mathcal{D}_{0}^{R, A}$ and $\Sigma^{K} \sim \lambda^{2}\left(\mathcal{D}_{0}^{K} * \mathcal{D}_{0}^{K}+\mathcal{D}_{0}^{R} * \mathcal{D}_{0}^{A}+\mathcal{D}_{0}^{A} * \mathcal{D}_{0}^{R}\right)$, where the star denotes convolution in the energy space.
}
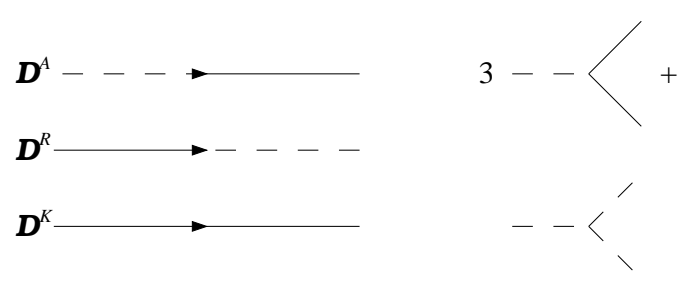

a)

FIG. 3 Example of the Keldysh diagrammatic for the $\phi^{3}$ theory. Fields $\phi_{c l}$ are denoted by full lines and $\phi_{q}$ by dashed lines. There are two vertices with relative coefficient 3. There is one retarded, one advanced and three Keldysh self-energy diagrams

Employing the "magic" identity $\operatorname{coth} \epsilon \operatorname{coth}(\epsilon+\omega)-1=\operatorname{coth} \omega(\operatorname{coth} \epsilon-\operatorname{coth}(\epsilon+\omega))$, one may immediately see that if $\mathcal{D}_{0}$ satisfy FDT, Eq. (17), then so does $\Sigma$, namely $\Sigma^{K}=\operatorname{coth} \frac{\beta \omega}{2}\left(\Sigma^{R}-\Sigma^{A}\right)$. Then the Dyson equation (34) says that the exact propagator also satisfy FDT. The "magic" identity is directly responsible for the fact that the Planck distribution (coth $\left.\frac{\beta \omega}{2}\right)$ nullifies the collision integral. 


$$
S_{\text {source }}=\int_{-\infty}^{\infty} d t\left[V_{c l}\left(\bar{\phi}_{c l} \phi_{q}+\bar{\phi}_{q} \phi_{c l}\right)+V_{q}\left(\bar{\phi}_{c l} \phi_{c l}+\bar{\phi}_{q} \phi_{q}\right)\right] .
$$

Notice that the quantum source is coupled to the classical component of the density (defined in a usual way as a half sum of the density on the forward and backward branches) and vise-versa. We define now the generating function as

$$
Z\left[V_{c l}, V_{q}\right]=\left\langle e^{i S_{\text {source }}}\right\rangle .
$$

The classical component of the source field is nothing but classical external scalar potential, which is supposed to be the same on the two branches. The presence of such field does not violate the fact that the forward and the backward evolution brings the system back to the initial state. Therefore it does not change the normalization $Z=1$. We therefore arrive to the conclusion

$$
Z\left[V_{c l}, V_{q}=0\right]=1 .
$$

One can also check this statement explicitly by e.g. expanding the action in powers of $V_{c l}$ and making contractions. All terms in this expansion vanish due to the fact that $\left\langle\phi_{q} \bar{\phi}_{q}\right\rangle=0$. Eq. (37) is a close relative of Eq. (20) and is just another manifestation of the causality. As a result, adding to the theory classical fields alone does not do any good we still work with unity. Therefore, one necessarily has to introduce quantum sources (they change sign by going from the forward to the backward branches of the contour). The presence of such source fields explicitly violates causality, and thus changes the partition (generating) function. On the other hand such fields usually do not have a physical meaning (see, however, the next section) and play only auxiliary role. In most cases one uses them only to generate observables (by appropriate differentiation) and then put them to zero, restoring the causality structure of the action. Notice that the classical component, $V_{c l}$, does not have to be taken to zero.

Let us see how it works. Suppose we are interested in the average density of bosons at time $t$ in presence of a certain scalar potential $V_{c l}(t)$. Of course, we are looking for the classical component of the density. According to Eqs. (35) and (36) it is given by

$$
n\left(t ; V_{c l}\right)=-\left.i \frac{\delta}{\delta V_{q}(t)} Z\left[V_{c l}, V_{q}\right]\right|_{V_{q}=0} .
$$

To calculate this quantity one has to find first an appropriate (classical) saddle point of the action in presence of $V_{c l}$ (as was mentioned above this amounts to solving the kinetic equation) and then study quantum corrections. The problem is simplified if the external field, $V_{c l}$, is weak in some sense. Then one may go away with the linear response

$$
\left.\chi^{R}\left(t, t^{\prime}\right) \equiv \frac{\delta}{\delta V_{c l}\left(t^{\prime}\right)} n\left(t ; V_{c l}\right)\right|_{V_{c l}=0}=-\left.i \frac{\delta^{2}}{\delta V_{c l}\left(t^{\prime}\right) \delta V_{q}(t)} Z\left[V_{c l}, V_{q}\right]\right|_{V_{q}=V_{c l}=0} .
$$

I put the subscript $R$, anticipating on the physical ground that the response function must be retarded. We shall demonstrate it momentarily. First, let us introduce for esthetical reasons the matrix

$$
\chi_{\alpha, \beta}\left(t, t^{\prime}\right) \equiv-\left.i \frac{\delta^{2}}{\delta V_{\beta}\left(t^{\prime}\right) \delta V_{\alpha}(t)} Z[V]\right|_{V=0}=\left(\begin{array}{cc}
0 & \chi^{A}\left(t, t^{\prime}\right) \\
\chi^{R}\left(t, t^{\prime}\right) & \chi^{K}\left(t, t^{\prime}\right)
\end{array}\right) .
$$

The fact that $\chi_{c l, c l}=0$ is obvious from Eq. (37). To evaluate the linear response matrix $\chi$ consider first the Gaussian action, Eq. (19). Adding the source term, Eq. (35), and integrating out the boson fields $\bar{\phi}, \phi$ one obtains

$$
Z[V]=e^{-\operatorname{Tr} \ln (1+\mathcal{D} \hat{V})},
$$

where $\hat{V}=\left(V_{q} \sigma_{0}+V_{c l} \sigma_{1}\right) / 2$. Notice that the normalization is just right, since $Z[0]=1$. One may now expand $\ln (1+\mathcal{D} \hat{V})$ to the second order in $\hat{V}$ and then differentiate twice. It is a straightforward matter to see that the causality structure of $\mathcal{D}$ imposes it also on $\chi$. E.g. $\chi^{R}\left(t, t^{\prime}\right) \sim \operatorname{Tr}\left\{\mathcal{D} \sigma_{1} \mathcal{D} \sigma_{0}\right\}=\mathcal{D}^{R}\left(t, t^{\prime}\right) \mathcal{D}^{K}\left(t^{\prime}, t\right)+\mathcal{D}^{K}\left(t, t^{\prime}\right) \mathcal{D}^{A}\left(t^{\prime}, t\right)$, which is obviously retarded. In presence of interactions, Eq. (21), one may perform Hubbard-Stratonovich transformation and then integrate out $\bar{\phi}, \phi$ fields. At this stage the causality structure of $\chi$ becomes apparent (it does not mean yet that one knows to evaluate $\chi$ ).

The purpose of the above discussion was to demonstrate that even for the linear response problems (which may be always solved in Matsubara technique) the Keldysh formulation is natural and transparent. For one thing it allows 
one to overcome the analytical continuation procedure, which may happen to be a headache. The other advantage is that it gives both response and correlation functions in the single framework 12 .

11. Counting statistics and counting fields. A very interesting application of the Keldysh formalism was suggested recently by L. S. Levitov and coworkers [11]. Suppose we are interested in a certain quantum mechanical observable $\hat{O}$ (for example, a total charge which passed through a wire during a long time $t_{f}-t_{i}$ ). One may look for e.g. its (quantum) expectation value, $\langle\hat{O}\rangle$, or noise $\left\langle\hat{O}^{2}\right\rangle-(\langle\hat{O}\rangle)^{2}$, or even higher order moments. The most general quantity, however, is the distribution function: $P(O)$ - probability to observe the value $O$ in a single measurement of $\hat{O}$. If one knows $P(O)$, then $\left\langle\hat{O}^{k}\right\rangle=\int d O P(O) O^{k}$. Instead of discussing $P(O)$ it is convenient to consider its Fourier transform $F(\lambda)=\int d O P(0) e^{i \lambda O}$. The variable $\lambda$ - is called the counting field, and $P(O)$ (or $F(\lambda)$ ) is called the counting statistics.

From the above expressions one may deduce that

$$
F(\lambda)=\left\langle e^{i \lambda \hat{O}}\right\rangle
$$

The non-trivial fact is that the angular brackets in this expression must be understood as the averaging along the Keldysh contour. The prove of this statement requires a careful consideration of the time-ordering convention for the higher moments of $\hat{O}$. We refer a reader to the original works [1],12] for more details. If the Keldysh nature of the averaging in Eq. (42) is accepted, it should be obvious by now that the counting field $\lambda$ is a purely quantum field. Indeed, $\lambda$ was introduced to generate moments of $\hat{O}$ upon differentiation. As we have seen in the previous section it is the quantum component of the source field (having different sign on forward and backward branches (!)), which serves for this purpose. As a result, Eq. (42) suggests that the Keldysh action of the system at hand must be modified as

$$
S^{\lambda}[\phi]=S[\phi]+\int_{-\infty}^{\infty} d t \lambda_{q}(t) \hat{o}_{c l}(t)
$$

We put $\hat{O}=\int_{t_{i}}^{t_{f}} d t \hat{o}_{c l}(t)$, where the classical observable $\hat{o}_{c l}(t)$ is expressed through the fields $\phi(t)$. We have also put $\lambda_{q}(t) \equiv \lambda \theta\left(t-t_{i}\right) \theta\left(t_{f}-t\right)$ - thus the name - counting field.

The novel feature is that the quantum source, $\lambda_{q}$, should not be taken to zero in the end of calculations. It rather remains finite and possibly even large to allow the inverse Fourier transform of $F(\lambda)$. The presence of the finite quantum source in the action spoils immediately the causality structure of the theory. For example, $S^{\lambda}\left[\phi_{q}=0\right] \sim$ $\lambda \neq 0$. As a result, $\Phi_{q}=0$ is not a saddle point solution any more. There are no classical saddle points in the theory ! A new non-classical saddle point has in general a non-zero action $S_{s p}=S_{s p}(\lambda) \neq 0$. Neglecting fluctuation effects, one finds for the generating function of the irreducible moments: $-i \ln F(\lambda)=S_{s p}(\lambda)$. Such program (including fluctuations) was realized by Yu. V. Nazarov [12] for the counting statistics of charge passing through a diffusive wire. See also related works on counting statistics of adiabatic pumps 22].

To conclude this section I want to mention a striking similarity between counting statistics in Keldysh theory and statistics of rare events in supersymmetric technique [23]. To my opinion this subject is far from being closed.

12. Keldysh technique in disordered systems. Due to the presence of the intrinsic normalization condition, $Z=1$, the Keldysh technique is ideally suited for treatment of disordered systems, such as spin glasses or dirty metals. Notice that the response and correlation functions were defined by differentiation of $Z[V]$, Eq. (40), rather than more familiar $\ln Z[V]$ (since $Z\left[V_{q}=0\right]=1$ this is completely equivalent). Let me also mention again that the normalization constant, $\operatorname{Tr}\left\{\rho_{0}\right\}$, introduced in Eq. (2), is disorder-independent. The point is that disorder is switched on (and off) on forward (backward) parts of the contour after (before) $t=-\infty$. As a result, the "partition" or generating function $Z$ (rather than its logarithm) is the proper object to be averaged over the quenched disorder. This circumvent the

\footnotetext{
${ }^{12}$ In the equilibrium response and correlation are connected by the FDT: $\chi^{K}(\omega)=\operatorname{coth} \frac{\beta \omega}{2}\left(\chi^{R}(\omega)-\chi^{A}(\omega)\right)$. However, out of the equilibrium they require separate calculations.
} 
need to introduce replica [24] (or supersymmetry 25) to take care of the logarithm. This was first realized in the context of classical spin glasses (MSRD version of Keldysh) by H. Sompolinsky [13] and substantially advanced later [14]. In the context of electrons in the random potential the corresponding theory (Keldysh non-linear $\sigma$-model) was developed in Refs. 15, 16, 26.

Here I restrict myself to a few remarks only. On the perturbative level the replica and supersymmetry methods are supposed to eliminate "parasitic" diagrams. Such decoupled closed loop diagrams are generated if one erroneously averages $Z$ instead of $\ln Z$. The replica trick first prescribes factor $n$ (number of replica) to such loops and then eliminates them by taking the limit $n \rightarrow 0$. In the supersymmetry bosonic loops exactly cancel fermionic ones. The Keldysh analog of such cancellation mechanism is given by Eqs. (13) and (14). Indeed, the closed loops coupled to the rest of a diagram by the static disorder carry either only retarded or only advanced Green functions ${ }^{13}$. The later vanish according to Eqs. (13) (14). Therefore it is not surprising that all perturbative results of the non-linear $\sigma$-model (including electron-electron interactions) are correctly and elegantly reproduced in the Keldysh version [15, 16].

Whether or not the Keldysh $\sigma$-model contains the non-perturbative effects (such as instantons) - is a hundred dollar question. I believe that the answer is positive. At least, it is possible to obtain the non-perturbative (though only asymptotic) part of the level statistics via the Keldysh $\sigma$-model [17. To this end, one has to analyze carefully non-classical (having a non-zero action) saddle points on the massless manifold of the $\sigma$-model. In any case, a lot to be done before the Keldysh technique will become a reliable tool for the non-perturbative calculations.

\section{DOI-PELITI TECHNIQUE FOR CLASSICAL SYSTEMS}

1. A bit of history. The technique was invented by Doi in 1976 [9] and later rediscovered by Peliti [10], and others [27,28]. J. Cardy and B. P. Lee were probably most persistent in applying the technique to various non-equilibrium statistical problems [29]. These notes are partially based on lectures of J. Cardy [30].

2. Motivation. We are talking about extremely broad and diverse types of problems whose applicability ranges from epidemiology and dynamics of biological populations to models of chemical reactions and glasses. The problems at hand are usually determined on some $d$-dimensional lattice. They are defined by specifying the following entities:

- The agents, usually named as $A, B, \ldots$ These may be molecules, animal species, stock options, vehicles, etc.

- Rules of agent's motion on the lattice. It may be e.g. random walk, or directed ballistic motion.

- Rules of agent's interactions. E.g. the simplest one is the binary annihilation: if two agents meet at the same lattice site they annihilate with probability $\mu$. The short way to write is

$$
A+A \stackrel{\mu}{\rightarrow} 0
$$

This model may, for example, describe a simple chemical reaction, where two identical molecules form a stable aggregate which segregates from the solution. An other famous example is the Lotka-Volterra system [31,32]

$$
\begin{aligned}
& A \stackrel{\mu}{\rightarrow} 0 ; \\
& B \stackrel{\sigma}{\rightarrow} 2 B ; \\
& A+B \stackrel{\lambda}{\rightarrow} 2 A,
\end{aligned}
$$

where $A$ designate "foxes" and $B$ - "rabbits". Foxes die with probability $\mu$; rabbits proliferate with probability $\sigma$, if a fox and a rabbit meet - the rabbit is eaten and another fox is produced with probability $\lambda$.

\footnotetext{
${ }^{13}$ Indeed, the static disorder $U_{d i s}$ enters the Keldysh matrix action as $U_{\text {dis }} \sigma_{1}$. Integrating out of the $\phi$ fields one ends up with $\operatorname{Tr} \ln \left(1+\mathcal{D} \sigma_{1} U_{\text {dis }}\right)$, cf. Eq. (41). Expanding the logarithm one finds terms of the form $\operatorname{Tr}\left\{\mathcal{D} \sigma_{1} \ldots \mathcal{D} \sigma_{1}\right\}$, the later are obviously $\operatorname{Tr}\left\{\mathcal{D}^{R} \ldots \mathcal{D}^{R}\right\}+\operatorname{Tr}\left\{\mathcal{D}^{A} \ldots \mathcal{D}^{A}\right\}=0$.
} 
3. Mean-field. The simplest way to treat systems described by Eqs. (44) or (45) is to employ the mean field equations. To this end one introduces the mean density of agents, say $A$, at a lattice site $r$ at time $t-n_{A}(r, t)$. For the system Eq. (44) with random walk motion (ARW - annihilating random walk) the mean field equation for the density is

$$
\frac{\partial n_{A}}{\partial t}=D \nabla_{r}^{2} n_{A}-\mu n_{A}^{2},
$$

where $D$ is the diffusion rate. Derivation of the mean field equation is self-evident from the definition of the model, Eq. (44). We shall see later, that from the perspective of Doi-Peliti technique the mean-field is nothing but equation for a classical saddle point. A reader is advised to write down and investigate the mean-field equations for the Lotka-Volterra system, Eq. (45).

The mean-field ignores fluctuations. It works reasonably well, when the number of agents at each site is large. In the opposite limit where the average number of agents on a site is less then unity, the system's behavior may be solely determined by fluctuation effects (especially in low spatial dimensionality). For example, Eq. (46) predicts $n_{A} \sim t^{-1}$ at $t \rightarrow \infty$, while the correct result [29,30] is $n_{A} \sim t^{-d / 2}$ for $d<2$ and $n_{A} \sim t^{-1}$ for $d>2$. The behavior is clearly very different from the mean-field prediction for dimensionality below the critical one, $d_{c}=2$. A traditional approximate way to handle fluctuations (which also stops short to obtain the above mentioned results) is by means of the Focker-Planck or Langevin equations. I shall comment below on the approximations involved in their derivation.

4. Master equation. The formally exact way to handle the problem is the master equation. One first defines a many-body microscopic configuration $\alpha$, determined by the (integer) number of agents at each lattice cite

$$
\alpha=\left\{n_{1}, n_{2}, \ldots n_{M}\right\},
$$

where $M=L^{d}$ is the total number of cites in the $d$-dimensional lattice. Next one defines $P(\alpha, t)$ - probability to find the system in the many-body configuration $\alpha$ at time $t$. The master equation has the form

$$
\frac{d}{d t} P(\alpha, t)=\sum_{\beta} \mathcal{R}_{\beta \rightarrow \alpha} P(\beta, t)-\sum_{\beta} \mathcal{R}_{\alpha \rightarrow \beta} P(\alpha, t),
$$

where the sums run over all many-body configurations $\beta$. The first term on the r.h.s. describes "in", while the second one "out" processes. The coefficients $\mathcal{R}_{\beta \rightarrow \alpha}$ and $\mathcal{R}_{\alpha \rightarrow \beta}$ are the transition rates. A particular case when,

$$
\frac{\mathcal{R}_{\beta \rightarrow \alpha}}{\mathcal{R}_{\alpha \rightarrow \beta}}=\frac{W(\beta)}{W(\alpha)},
$$

where $W$ is a certain positive function uniquely defined for each state, is said to satisfy the detailed balance condition.

As an example let us consider the binary annihilation on a single site. The microscopic state is specified by a positive integer number of agents, $\alpha=\{n\}$. According to Eq. (44) the master equation takes the form

$$
\frac{d}{d t} P(n, t)=\mu \frac{(n+2)(n+1)}{2} P(n+2, t)-\mu \frac{n(n-1)}{2} P(n, t) .
$$

The only non-zero rates are $\mathcal{R}_{n+2 \rightarrow n}$ and $\mathcal{R}_{n \rightarrow n-2}$, which are proportional to the number of possible pairs in the corresponding state. These rates do not satisfy the detailed balance.

5. Quantization. The basic idea [9] is to draw the analogy between the master equation and the many-body Schrödinger equation. Indeed, both equations are linear in $P$ (or $\Psi$ ) and first order in $\partial_{t}$. To make the analogy explicit one introduces the "second quantization". Define the ket-vector $|n\rangle$ as the microscopic state with $n$-agents. Let us also define vector

$$
|\Psi(t)\rangle \equiv \sum_{n=0}^{\infty} P(n, t)|n\rangle .
$$

Note that the weight, $P$, is probability rather than the amplitude. In these notations the master equation takes the form 


$$
\frac{d}{d t}|\Psi(t)\rangle=\sum_{n=0}^{\infty} \frac{d P(n, t)}{d t}|n\rangle=\frac{\mu}{2} \sum_{n=0}^{\infty}[(n+2)(n+1) P(n+2, t)-n(n-1) P(n, t)]|n\rangle .
$$

The aim now is to write the r.h.s. of this expression as $-\hat{H}|\Psi(t)\rangle$, where $\hat{H}$ is the "Hamiltonian" operator. To this end we introduce the creation and annihilation operators:

$$
\begin{aligned}
a^{\dagger}|n\rangle & =|n+1\rangle ; \\
a|n\rangle & =n|n-1\rangle .
\end{aligned}
$$

As a byproduct, one has $a|0\rangle=0$. One may immediately check that such operators are "bosonic" 四

$$
\left[a, a^{\dagger}\right]=1 .
$$

As for any pair of operators satisfying Eq. (55) one may prove the identity

$$
e^{a} f\left(a, a^{\dagger}\right)=f\left(a, a^{\dagger}+1\right) e^{a},
$$

where $f$ is an arbitrary operator-value function. With these definitions one may check that the Hamiltonian, we are looking for, has the form

$$
\hat{H}=\frac{\mu}{2}\left(\left(a^{\dagger}\right)^{2} a^{2}-a^{2}\right),
$$

where the first term on the r.h.s. is the "out" and the second one is "in" term. Generalization for the many site problem with the random walk (ARW) is straightforward, the resulting Hamiltonian is

$$
\hat{H}=D \sum_{<i j>}\left(a_{i}^{\dagger}-a_{j}^{\dagger}\right)\left(a_{i}-a_{j}\right)+\frac{\mu}{2} \sum_{j}\left(\left(a_{j}^{\dagger}\right)^{2}-1\right) a_{j}^{2},
$$

where $\langle i j\rangle$ are nearest neighbors.

6. Observables. So far we succeed to put the master equation into the Schrödinger form

$$
\frac{d}{d t}|\Psi(t)\rangle=-\hat{H}|\Psi(t)\rangle .
$$

The differences with the true Schrödinger equation are:

- There is no imaginary unity, meaning that we are dealing with the imaginary time Schrödinger equation.

- $\hat{H}$ is, in general, non-Hermitian (unless the detailed balance condition is satisfied).

- The most important: the mean value of some observable $\hat{O}$, is not $\langle\Psi(t)|\hat{O}| \Psi(t)\rangle$. Indeed, such combination is quadratic in $P$, while the mean value should be linear in probability, $P$.

To obtain the proper mean value one defines the bra-coherent state

$$
\left\langle\Psi_{0}\right| \equiv\langle 0| e^{a} .
$$

This state has the special property: $\left\langle\Psi_{0} \mid n\right\rangle=1$ for any $n$. This fact may be checked using Eq. (56). Employing this coherent state, one may show that the mean value of an observable may be written as

$$
\langle\hat{O}\rangle=\left\langle\Psi_{0}|\hat{O}| \Psi(t)\right\rangle .
$$

Indeed, let us consider density as an example, $\hat{O}=a^{\dagger} a$

\footnotetext{
${ }^{14}$ In problems where not more than one agent is allowed on a given site, (e.g. traffic flow models) one may probably employ "fermions". I am not aware, however, of such attempts.
} 


$$
\langle n(t)\rangle=\left\langle\Psi_{0}\left|a^{\dagger} a\right| \Psi(t)\right\rangle=\left\langle\Psi_{0}|a| \Psi(t)\right\rangle=\left\langle 0\left|e^{a} a^{\dagger} a \sum_{n} P(n, t)\right| n\right\rangle=\sum_{n} n P(n, t),
$$

in agreement with the expectations based on the meaning of $P(n, t)$. The second equality on the r.h.s. is very important; it is an immediate consequence of the coherent state being the left eigenstate of the creation operator, $\left\langle\Psi_{0}\right| a^{\dagger}=\left\langle\Psi_{0}\right|$.

7. Normalization. To have a consistent theory, one has to worry that the total probability to find the system in some state is unity

$$
1=\sum_{n} P(n, t)=\left\langle\Psi_{0} \mid \Psi(t)\right\rangle
$$

The last equality is just the mean value of the unit operator. One may solve formally the Schrödinger equation and write $|\Psi(t)\rangle=\exp \left\{-\hat{H}\left(a^{\dagger}, a\right) t\right\}|\Psi(0)\rangle$. Eq. (63) is satisfied iff $\left\langle\Psi_{0}\right| \hat{H}\left(a^{\dagger}, a\right)=0$. Since the coherent state is an eigenstate of the creation operator, $\left\langle\Psi_{0}\right| a^{\dagger}=\left\langle\Psi_{0}\right|$, one arrives at the conclusion that any legitimate Hamiltonian must obey

$$
\hat{H}\left(a^{\dagger}=1, a\right)=0 .
$$

E.g. the Hamiltonian of ARW, Eq. (58), indeed satisfy this condition. To introduce the functional integral representation we'll need to have the normal ordering of creation and annihilation operators. Therefore it is a good idea to commute $e^{a}$ through the Hamiltonian employing Eq. (56).

$$
\left\langle 0\left|e^{a} e^{-\hat{H}\left(a^{\dagger}, a\right) t}\right| \Psi(0)\right\rangle=\left\langle 0\left|e^{-\hat{H}\left(a^{\dagger}+1, a\right) t} e^{a}\right| \Psi(0)\right\rangle .
$$

If an observable is considered, the same commutation may be performed provided that the observable is written in terms of annihilation operators only. An example, of this procedure is provided by the second equality in Eq. (62). We shall redefine from now on the Hamiltonian as $H\left(a^{\dagger}, a\right) \equiv \hat{H}\left(a^{\dagger}+1, a\right)$. In terms of the new Hamiltonian the fundamental normalization condition, Eq. (63), is satisfied iff

$$
H\left(a^{\dagger}=0, a\right)=0 .
$$

We shall see momentarily that this condition is the direct analog of the Keldysh normalization condition, Eq. (20).

8. Field theory. Consider the "partition function" of the theory

$$
Z \equiv\left\langle 0\left|e^{-H\left(a^{\dagger}, a\right) t}\right| \tilde{\Psi}(0)\right\rangle
$$

where $|\tilde{\Psi}(0)\rangle=e^{a}|\Psi(0)\rangle$ is the renormalized initial microscopic state, its precise form is not very important for our further discussion. Due to normalization condition and according to Eqs. (63), (65), $Z=1$. We divide now the time interval $[0, t]$ onto $N \rightarrow \infty$ slices and introduce the coherent state resolution of unity at each time slice. As a result, one obtains in the standard way [19]

$$
Z=\int \mathcal{D} \phi^{*} \phi \exp \left\{-S\left[\phi^{*}(t), \phi(t)\right]\right\}
$$

where

$$
S\left[\phi^{*}(t), \phi(t)\right]=\int_{0}^{t} d t\left[\phi^{*} \partial_{t} \phi+H\left(\phi^{*}, \phi\right)\right] .
$$

For example, in the case of ARW one has

$$
S\left[\phi^{*}(t, r), \phi(t, r)\right]=\int_{0}^{t} d t \int d r\left[\phi^{*}\left(\partial_{t}-D \nabla_{r}^{2}\right) \phi+\frac{\mu}{2}\left(\left(\phi^{*}\right)^{2} \phi^{2}+2 \phi^{*} \phi^{2}\right)\right] .
$$


9. Analogy with the Keldysh technique. Notice that both in Keldysh and Doi-Peliti techniques we deal with the "partition function" $Z=1$. (No matter that the reasons for this normalization are very different: closed time contour in Keldysh versus conservation of probability in Doi-Peliti.) In both cases we succeed to write the partition function as the coherent state functional integral, cf. Eq. (3) and Eq. (68). To this end in both cases we had to double number of degrees of freedom: classical and quantum in Keldysh versus $\phi$ and $\phi^{*}$ in Doi-Peliti ${ }^{15}$. The normalization requires the strong condition on an acceptable action: Eq. (20) in Keldysh versus

$$
S\left[\phi^{*}=0, \phi\right]=0
$$

in Doi-Peliti. This equation is a direct consequence of Eqs. (66) and (69). Comparing Eqs. (20) and (71) one may suspect the analogy

$$
\phi^{*} \sim \phi_{q} ; \quad \phi \sim \phi_{c l}
$$

We shall see below that this analogy indeed works for all intends and purposes. Let us rewrite the quadratic part of the action of the ARW model in the following form

$$
S\left[\phi^{*}, \phi\right]=\frac{1}{2} \int d t\left(\phi, \phi^{*}\right)\left(\begin{array}{cc}
\underbrace{\partial_{t}-D \nabla_{r}^{2}}_{R} & \overbrace{-\partial_{t}-D \nabla_{r}^{2}}^{A} \underbrace{X}_{K}
\end{array}\right)\left(\begin{array}{c}
\phi \\
\phi^{*}
\end{array}\right) .
$$

Here $R$ and $A$ designate retarded and advanced linear classical operators. Symbol $K$ stays for the Keldysh component of the correlator. In our simplistic example it is absent, $X=0$. This fact is not generic by any means: in more complicated models e.g. Lotka-Volterra there is non-zero Keldysh component. Moreover, even if it is zero in the bare action - it is usually generated in the process of renormalization. On the other hand, the fact that the $\phi-\phi$ element is zero is not accidental. This zero is direct a consequence of Eq. (71) and it is protected against any renormalization.

Once the structure of the quadratic action is understood, one may write down propagators to build the perturbation theory (cf. Eq. (11) )

$$
\begin{aligned}
\left\langle\phi(t) \phi^{*}\left(t^{\prime}\right)\right\rangle & =\mathcal{D}^{R}\left(t, t^{\prime}\right) \sim\left(D q^{2}-i \omega\right)^{-1} \\
\left\langle\phi^{*}(t) \phi\left(t^{\prime}\right)\right\rangle & =\mathcal{D}^{A}\left(t, t^{\prime}\right) \sim\left(D q^{2}+i \omega\right)^{-1} \\
\left\langle\phi^{*}(t) \phi^{*}\left(t^{\prime}\right)\right\rangle & =0 \\
\left\langle\phi(t) \phi\left(t^{\prime}\right)\right\rangle & =\mathcal{D}^{K}\left(t, t^{\prime}\right) .
\end{aligned}
$$

As in the Keldysh case, $\mathcal{D}^{R}(t, t)=\mathcal{D}^{A}(t, t)=0$. It is exactly the same causality structure, which is responsible for the conservation of probability (normalization) and the entire internal consistency of the theory. The analogy between the two techniques goes actually much further than the perturbative expansion.

11. Saddle point equations. Exactly as in the case of Keldysh, the saddle point equation

$$
\frac{\delta S}{\delta \phi}=0
$$

may be always solved by

$$
\Phi^{*}=0
$$

The saddle point solutions are denoted by capital $\Phi$. Under the condition Eq. (76) the second saddle point equation takes the form

\footnotetext{
${ }^{15}$ Notice that since we are interested in real density of agents, one may naively expect to obtain a field theory of the single real bosonic field. We have to deal with both $\phi$ and $\phi^{*}$, however, despite of the fact that the object is purely classic and the phase has no evident meaning.
} 


$$
\frac{\delta S}{\delta \phi^{*}}=\mathcal{O}^{R}[\Phi] \Phi=0,
$$

where $\mathcal{O}^{R}[\Phi]$ is the retarded operator. In the example of ARW, Eq. (70)

$$
\mathcal{O}^{R}[\Phi]=\partial_{t}-D \nabla_{r}^{2}+\mu \Phi
$$

and the saddle point equation (77) is nothing but the mean-field equation, Eq. (46). Notice, that the classical (meanfield) equation of motion is a result of variation over the "quantum" $\left(\phi^{*}\right)$ component (in the limit where the later is zero). In view of Eqs. (71) and (76), the action on any classical solution is zero.

The existence and role of "non-classical" saddle points (with $\Phi^{*} \neq 0$ and $S_{s p} \neq 0$ ) in the context of Doi-Peliti technique, to the best of my knowledge, was not yet investigated.

12. Semiclassical approximation. To include fluctuation effects in the semiclassical approximation one keeps terms up to the second order in $\phi^{*}$ in the action. For the ARW problem the entire action is semiclassical (this is not generic by any means). Let us rewrite it in the following generic semiclassical form

$$
S_{s c l}=\int d t\left[\frac{1}{2} \phi^{*}\left[\mathcal{D}^{-1}\right]^{K} \phi^{*}+\phi^{*} \mathcal{O}^{R}[\phi] \phi\right] .
$$

For the ARW problem $\left[\mathcal{D}^{-1}\right]^{K}=\mu \phi^{2}>0$. Positive definiteness of this quantity is necessary for the convergence of the $\phi^{*}$ integral. From this point one may again proceed in two directions:

(i) Since the action Eq. (79) is Gaussian in $\phi^{*}$ one may integrate it out and end up with the theory of the single filed, $\phi$. The corresponding action is practically identical to Eq. (28). Employing the transfer-matrix method, one arrives at the Focker-Planck equation.

(ii) One may perform the Hubbard-Stratonovich transformation with the auxiliary stochastic field $\xi(t)$ to decouple the term quadratic in $\phi^{*}$. Subsequent integration over $\phi^{*}$ results in the functional $\delta$-function. This way one obtains stochastic Langevin equation

$$
\mathcal{O}^{R}[\phi] \phi(t)=i \xi(t),
$$

where $\xi(t)$ is Gaussian noise with the correlator

$$
\left\langle\xi^{2}(t)\right\rangle=\left[\mathcal{D}^{-1}\right]^{K} .
$$

Some studies start from postulating Langevin (or Focker-Planck) dynamics for problems at hand. We note that, this approach is not exact, but rather a semiclassical approximation. In some cases, like the ARW, the semiclassical approximation and hence the Langevin dynamics is indeed exact. However, even in such cases the correlator of noise is far from being trivial. Moreover, the noise term in Langevin equation, Eq. (80), contrary to any intuition, happens to be pure imaginary. It is hard to imagine how such dynamics could be postulated from the outset.

13. Sources. To calculate an observable, e.g. mean density, one need to introduce sources. As was mentioned above, mean value of any observable may be expressed through annihilation operators only, c.f. the second equality in Eq. (62). In the functional integral language it means that pre-exponential factors at hand are given in terms of the "classical" field, $\phi$, only. Therefore the source term, one needs, has the form $\int d t V^{*}(t) \phi(t)$ and the generating functional is given by

$$
Z\left[V^{*}\right]=\left\langle e^{\int d t V^{*}(t) \phi(t)}\right\rangle,
$$

where the angular brackets stay for averaging with the action, Eq. (69). With this generating functional one may obtain, e.g., the mean density of agents

$$
\langle n(t)\rangle=\left\langle\Psi_{0}|a| \Psi(t)\right\rangle=\int \mathcal{D} \phi^{*} \phi e^{-S\left[\phi^{*}(t), \phi(t)\right]} \phi(t)=\left.\frac{\delta}{\delta V^{*}(t)} Z\left[V^{*}\right]\right|_{V^{*}=0} .
$$


Other observables are evaluated in the similar fashion. Note that as in the Keldysh technique, observables are given in terms of the "classical" field, $\phi$, while the generating function is functional of the fictions "quantum" source, $V^{*}(t)$, which is to be taken to zero after differentiation.

14. Short summary. The aim of this chapter is not to give a detailed account of a specific calculation employing the Doi-Peliti technique. Lectures and papers of J. Cardy et al. [30,29] are highly recommended for this purpose. My intention was to highlight the similarities and common concepts with the Keldysh techniques. The hope is to facilitate intermixing of the two largely non-intercepting communities. Let me stress once again common points of the two techniques:

- Internal fundamental normalization, $Z=1$.

- Doubling of degrees of freedom: "classical-quantum".

- Causality: retarded-advanced structure of the action and the propagators.

- Existence of the classical extrema: $\Phi_{q}=0 ; \mathcal{O}^{R} \Phi_{c l}=0$.

- Langevin and Focker-Planck approaches as the semiclassical approximation - second order in $\phi_{q}$.

- Generating function of classical observables is functional of the quantum source.

I am indebted to V. Lebedev, A. Andreev, A. Altland and V. Elgart for teaching me various aspects of the techniques and to L. V. Keldysh, M. Doi and L. Peliti for inventing them. This research was supported in part by the BSF grant N 9800338.

[1] L. V. Keldysh, Zh. Eksp. Teor. Fiz. 47, 1515 (1964); [Sov. Phys. JETP 20, 1018 (1965)].

[2] J. Schwinger, J. Math. Phys. 2, 407 (1961).

[3] R. P. Feynman and F. L Vernon Jr., Ann. Phys. 24, 118 (1963).

[4] E. M. Lifshitz, and L. P. Pitaevskii, Statistical Physics, part II, Pergamon Press (1980).

[5] G. D. Mahan, Many-particle physics, Plenum Press, NY, 1990.

[6] J. Rammer, and H. Smith, Rev. Mod. Phys. 58, 323, (1986).

[7] H. W. Wyld, Ann. Phys., 14, 143 (1961).

[8] P. C. Martin, E. D. Siggia, and H. A. Rose, Phys. Rev. A 8, 423 (1973); DeDominics, J. Physique (Paris), 37, C1 (1976).

[9] M. Doi, J. Phys. A 9, 1465 (1976).

[10] L. Peliti, J. Physique 46, 1469 (1985).

[11] L. S. Levitov, and G. B. Lesovik, JETP Lett., 58, 230 (1993); L. S. Levitov, H. Lee, and G. B. Lesovik, J. Math. Phys. 37, 4845 (1996); D. A. Ivanov, H. W. Lee, and L. S. Levitov, Phys. Rev. B 56, 6839 (1997).

[12] Yu. V. Nazarov, Ann. Phys. (Leipzig), 8, 507 (1999); W. Belzig, Yu. V. Nazarov, Phys. Rev. Lett. 87, 067006 (2001); Yu. V. Nazarov, M. Kindermann, cond-mat/0107133.

[13] H. Sompolinsky, Phys. Rev. Lett 47, 935 (1981); H. Sompolinsky, and A. Zippelius, Phys. Rev. B 25, 6860 (1982).

[14] V. S. Dotsenko, M. V. Feigelman, and L. B. Ioffe, Spin glasses and related problems, v. 15, pt. 1 Soviet scientific reviews 15 (Harwood Academic, New-York 1990); L. F. Cugliandolo, and G. Lozano, cond-mat/9807138.

[15] A. Kamenev, and A. Andreev, Phys. Rev. B 60, 2218, (1999).

[16] C. Chamon, A. W. W. Ludwig, and C. Nayak, Phys. Rev. B 60, 2239, (1999).

[17] A. Altland and A. Kamenev, Phys. Rev. Lett., 855615 (2000).

[18] A. A. Abrikosov, L. P. Gorkov, I. E. Dzyaloshinski, Methods of quantum field theory in statistical physics, Dover, NY, 1963.

[19] J.W. Negele and H. Orland, Quantum Many-Particle Systems, Adelison-Wesley, 1988.

[20] L. B. Ioffe, D. Sherrington, cond-mat/9709270; A. V. Lopatin, L. B. Ioffe, cond-mat/9904219.

[21] Sh. M. Kogan, A. Ya. Shulman, Zh. Eksp. Teor. Fiz. 56, 862 (1969) [Sov. Phys, JETP 29, 467 (1969)].

[22] A. V. Andreev and A. Kamenev, Phys. Rev. Lett. 85, 1294 (2000); L.S. Levitov, cond-mat/0103617; A. V. Andreev, E. G. Mishchenko, cond-mat/0104211.

[23] B. A. Muzykantskii and D. E. Khmelnitskii, Pisma Zh. Eksp. Teor. Fiz. 62, 68 (1995) [JETP Lett. 62, 76 (1995)].

[24] S. F. Edwards and P. W. Andreson, J. Phys. F. 5, 89 (1975).

[25] K. B. Efetov, Adv. Phys. 32, 53 (1983); K. B. Efetov, Supersymmetry in Disorder and Chaos, Cambridge University Press, 1997.

[26] M. V. Feigel'man, A. I. Larkin, M. A. Skvortsov, Phys. Rev. B 61 , 12361 (2000).

[27] D. C. Mattis and M. L. Glasser, Rev. Mod. Phys. 70, 979 (1998).

[28] P. Grassberger and M. Scheunert, Fortschr. Phys. 28, 547 (1980). 
[29] B. P. Lee, J. Phys. A 27, 2633 (1994); B. P. Lee and J. Cardy. Phys. Rev. E 50, 3287 (1994); B. P. Lee and J. Cardy, J. Stat. Phys. 80, 971 (1995).

[30] J. Cardy, http://www-thphys.physics.ox.ac.uk/users/JohnCardy/home.htm]

[31] A. J. Lotka, Proc. Natl. Acad. Sci. USA 6, 410 (1920); V. Volterra, Lecon sur la Theorie Mathematique de la Lutte pour le via. (Gauthier-Villars, Paris, 1931).

[32] E. Bettelheim, O. Agam, and N. M. Shnerb, cond-mat/9908450. 